\title{
Artifacts-reduced 2D cine SSFP with flow compensation for myocardial BOLD imaging
}

\author{
Xiangzhi Zhou*1, Sotirios A Tsaftaris ${ }^{1}$, Ying Liu ${ }^{1}$, Richard Tang1, \\ Rachel Klein ${ }^{1}$, Sven Zuehlsdorff ${ }^{2}$, Debiao Li ${ }^{1}$ and Rohan Dharmakumar ${ }^{1}$
}

Address: ${ }^{1}$ Northwestern University, Chicago, IL, USA and ${ }^{2}$ Siemens Medical Solutions USA, Inc., Chicago, IL, USA

* Corresponding author

from 13th Annual SCMR Scientific Sessions

Phoenix, AZ, USA. 21-24 January 2010

Published: 21 January 2010

Journal of Cardiovascular Magnetic Resonance 20 I0, I2(SuppI I):P68 doi: 10.1 I86/I532-429X-I2-SI-P68

This abstract is available from: http://jcmr-online.com/content/I2/SI/P68

(c) 2010 Zhou et al; licensee BioMed Central Ltd.

\section{Introduction}

Robust image quality is critical for reliable detection and evaluation of myocardial oxygenation changes with blood-oxygen-level-dependent (BOLD) imaging. Recently, balanced SSFP methods have been employed to overcome image quality limitations associated with myocardial BOLD methods. However, the long TRs required for BOLD contrast, can lead to unwanted flow/motion artifacts, ultimately compromising image quality.

\section{Purpose}

To minimize flow/motion artifacts in cardiac phaseresolved SSFP BOLD imaging.

\section{Methods}

Six dogs were studied in a $1.5 \mathrm{~T}$ scanner using first-order flow/motion-compensated SSFP method over the whole left ventricle. Basal, mid-ventricular, and apical images were acquired in cine mode with three different approaches: (A) TR $=3.5 \mathrm{~ms}$ (conventional); (B) TR $=6.2$ $\mathrm{ms}$ (without compensation); and (C) TR $=6.2 \mathrm{~ms}$ (with flow/motion compensation). Three experts used (i) Ghost artifacts (GA), an impression of artifacts observed within the image; and (ii) myocardial inhomogeneity (MI), a measure of the signal homogeneity within the left-ventricular myocardium, to evaluate myocardial signal characteristics. One-way ANOVA was performed to ascertain whether there were any differences in the indices with various approaches.
Preliminary myocardial BOLD studies were performed on animals $(\mathrm{n}=2)$ with controllable LCX stenosis to evaluate the benefits of flow compensation. Studies were performed under rest and adenosine stress in the absence or presence of severe (80-90\%) stenosis. Other scan parameters were: matrix $=126 \times 192 ; \mathrm{FOV}=157.5 \mathrm{~mm} \times 240$ $\mathrm{mm}$; slice-thickness $=5 \mathrm{~mm} ; \mathrm{TE}=3.1 \mathrm{~ms}$; and flip-angle $=$ $70^{\circ}$.

\section{Results}

Mean scores assessed over the various positions along the left ventricle over all animals using the 3 approaches are summarized in Figure 1. The scores of Approach (B) were significantly higher than Approaches A and C $(\mathrm{p}<0.01)$. For TR $=6.2 \mathrm{~ms}$, GA and MI increased when moving slice position from the apex to base. There was no statistical difference in the scores associated with Approaches (A) and (C). Figure 2 shows a representative set of late-systolic and late-diastolic mid-ventricular images obtained from a dog under baseline and severe LCX stenosis (SS). With stenosis, the signal loss in the LCX territories became apparent; consistent with the expectation that stenoses lead to significant reductions in myocardial oxygen reserve. Results showed remarkable reduction in image artifacts, permitting the visualization of signal loss in the LCX territories over the entire cardiac cycle in the presence of SS.

\section{Conclusion}

First-order flow/motion compensation strategy employed in this study provides significant improvement in image 

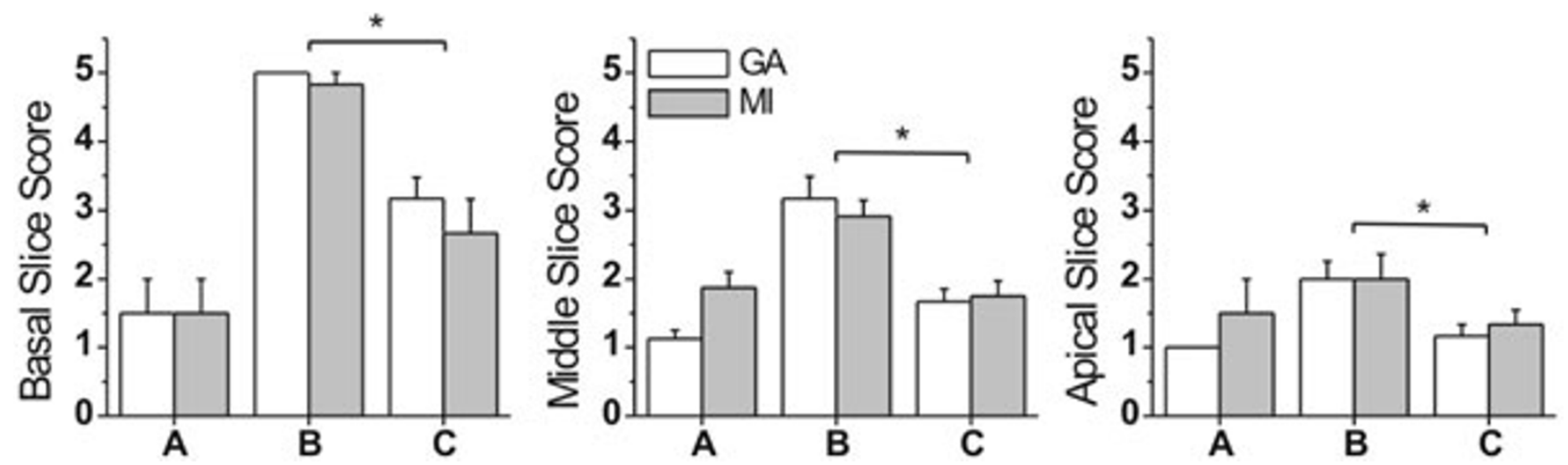

Figure I

Expert scores (I for best, 5 for worst) for ghost artifacts (GA) and myocardial inhomogeneity (MI) for the basal, middle (mid-ventricular), and apical slices acquired with 3 SSFP sequences A: TR = 3.5 ms, B: TR-239 ms, (A and B acquired without flow compensation): and C: TR = $6.2 \mathrm{~ms}$ (with flow compensation). Ghost artifacts and myocardial inhomoegeneity in images acquired with Approach B are significantly higher than in images acquired with Approach C $(p<0.0 \mathrm{I})$ in all 3 slices. For TR-6.2 ms $(B, C)$, GA are Ml increase as slice position moves form apex to base. Note that there is no significant difference in GA or MI for images acquired with Approach $A$ and $C$ in the basal, middle or apical alice.

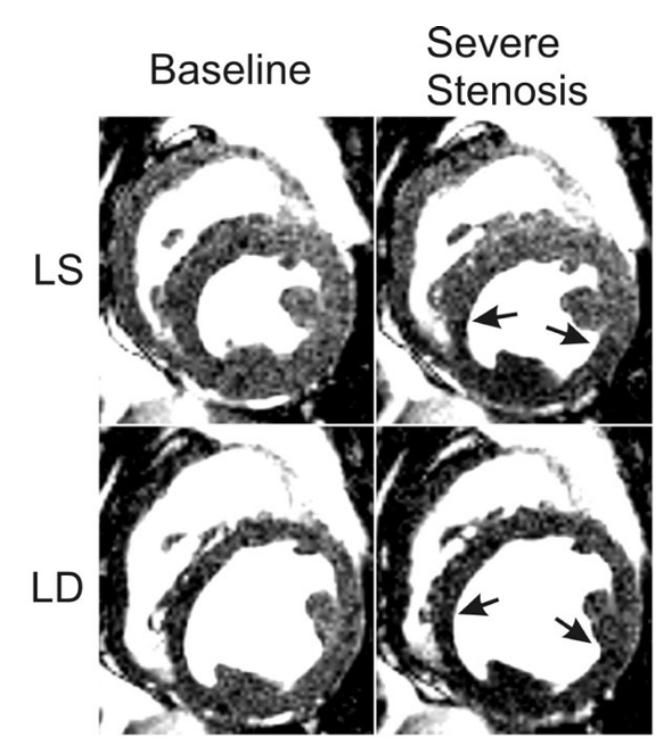

Figure 2

Representative cardiac phase-dependent short-axis mid-LV images [upper row: late-systole (LS); lower row: late-diastole (LD)] obtained from a dog model with no coronary artery stenosis under rest (left column), with severe LCX stenosis under adenosine stress (right column(using flow-compensated 2D SSFP sequence. The scan parameters were: $T R=6.2 \mathrm{~ms}$, $F A=70^{\circ}, T_{R E S}=12 \mathrm{~ms}$. Note the discriminating signal loss in the LCX territories marked by arrows (short arc) in the presence of LCX stenosis. quality compared to the standard cine BOLD-SSFP MRI. The method proposed here may enable a more reliable means for evaluating BOLD signal changes over the entire cardiac cycle. 